\title{
The Association between Cigarette Smoking and Efavirenz Plasma Concentration using the Population Pharmacokinetic Approach
}

\author{
Ngah Kuan Chow ${ }^{1,2}$, E-Jinq Wong ${ }^{3}$, Lee Lee Low ${ }^{3}$, Siti Maisharah Sheikh Ghadzi ${ }^{1}$, \\ Sabariah Noor Harun ${ }^{1}$, and Amer Hayat Khan ${ }^{1}$ \\ ${ }^{1}$ Universiti Sains Malaysia \\ ${ }^{2}$ Hospital Kulim \\ ${ }^{3}$ Hospital Sultanah Bahiyah
}

November 30, 2020

\begin{abstract}
Aim: Efavirenz is still widely used as the preferred first-line antiretroviral agent in the middle- and low- income countries, including Malaysia. The efavirenz population pharmacokinetic profile among HIV-positive smokers is still unknown. We aimed to assess the association of smoking with efavirenz and the differences in HIV clinical outcomes. Methods: A total of 154 stable HIV-positive patients on efavirenz in northern Malaysia were recruited with a sparse sampling for this multicentre prospective cohort study. The association between smoking and efavirenz pharmacokinetic parameters was determined using the non-linear mixed-effect model (NONMEM). A mixture model of clearance was adopted to describe the metaboliser status because genetic data is unavailable. The effect of smoking on HIV clinical markers (CD4, CD4 / CD8 ratio and viral blips) for at least two years after the antiretroviral initiation was also investigated. Results: Our data were best fitted with a one-compartment mixture model with first-order absorption without lag time. Smoking significantly associated with higher clearance (CL/F) $(\beta=1.39$; 95\% confidence interval (CI): 1.07 to 1.91), while weight affected both CL/F and volume (V/F). From the mixture model, $20 \%$ of patients were in the slow clearance group, which mimic the genotype distribution of slow metaboliser. An efavirenz dose reduction is not recommended for smokers [?]60kg with normal metabolism rate. Smoking significantly associated with slower normalisation of CD4 and CD4 / CD8 ratio. Conclusion: HIV-positive smokers presented with significantly higher efavirenz clearance and unfavourable clinical outcomes. Close monitoring of adherence and clinical response among smokers is warranted.
\end{abstract}

The Association between Cigarette Smoking and Efavirenz Plasma Concentration using the Population Pharmacokinetic Approach

Ngah Kuan Chow ${ }^{1,2}$, E-Jinq Wong ${ }^{3}$, Lee Lee Low $^{4}$, Siti Maisharah Sheikh Ghadzi ${ }^{1}$, Sabariah Noor Harun $^{1}$, Amer Hayat Khan ${ }^{1}$

${ }^{1}$ Discipline of Clinical Pharmacy, School of Pharmaceutical Sciences, Universiti Sains Malaysia, Penang, Malaysia

${ }^{2}$ Department of Pharmacy, Hospital Kulim, Kedah, Malaysia

${ }^{3}$ Department of Pharmacy, Hospital Sultanah Bahiyah, Kedah, Malaysia

${ }^{4}$ Department of Medicine, Hospital Sultanah Bahiyah, Kedah, Malaysia

\section{Corresponding Author}

Dr Amer Hayat Khan 
Discipline of Clinical Pharmacy, School of Pharmaceutical Sciences, Universiti Sains Malaysia, 11800 Penang, Malaysia.

Email: dramer2006@gmail.com

Chow Ngah Kuan

Discipline of Clinical Pharmacy, School of Pharmaceutical Sciences, Universiti Sains Malaysia, 11800 Penang, Malaysia.

Email: kimi_nk_chow@yahoo.com

\section{Author contributions}

CNK was responsible for study conception and design, collecting and analysing the data and drafting the first manuscript. AHK participated in material preparation, review and edited the manuscript. SNH participated in concept development, reviewed and supported the analyses, reviewed and edited the manuscript. SMSG reviewed and supported the analyses, reviewed and edited the manuscript. WEJ and LLL contributed ideas to the clinical aspect of the data and reviewed the manuscript. All authors read and approved the final manuscript.

\section{Declarations}

Funding and conflict of interest:

No funding was received for this study. All authors declare that they have no conflicts of interest or financial relationships relevant to this article to disclose.

Consent to participate:

Informed consent was obtained from all individual participants included in the study.

Consent to publish:

All participants signed informed consent regarding publishing their data.

Ethics approval:

This study has been granted ethical approval from the Medical Research and Ethics Committee (MREC), Ministry of Health Malaysia (ID: NMRR-18-445-40064) and the Human Research Ethics Committee (JEPeM), Universiti Sains Malaysia (Code: USM/JEPeM/20030178), which complies with the Declaration of Helsinki.

Principal Investigator:

The authors confirm that the principal investigator for this paper is Ngah Kuan Chow and that she had direct clinical responsibility for patients.

\section{Running head}

Smoking \& Efavirenz - Pop PK Approach

Word count: 3981

Table count: 3

Figure count: 5

\section{What is already known about this subject:}

- In vitro studies suggested that cigarette smoke induces CYP2B6. However, the efavirenz pharmacokinetic profile among HIV-positive smokers is unknown.

- Efavirenz is recommended to be given at a lower dose of 400mg daily. 
- CYP2B6 genotype is essential in determining efavirenz population pharmacokinetic parameters.

\section{What this study adds:}

- Clearance of efavirenz is higher among HIV-positive smokers regardless of their genotypes.

- Efavirenz $400 \mathrm{mg}$ daily or lower is not recommended for smokers with normal efavirenz metabolism rate and weigh more than $60 \mathrm{~kg}$.

- When genetic data is not available, the population efavirenz clearance can be estimated using the mixture model approach.

\section{Hosted file}

EFV main text BJCP.pdf available at https://authorea.com/users/378396/articles/494917-theassociation-between-cigarette-smoking-and-efavirenz-plasma-concentration-using-thepopulation-pharmacokinetic-approach

\section{Hosted file}

Table 1 BJCP.pdf available at https://authorea.com/users/378396/articles/494917-theassociation-between-cigarette-smoking-and-efavirenz-plasma-concentration-using-thepopulation-pharmacokinetic-approach

\section{Hosted file}

Table 2 BJCP.pdf available at https://authorea.com/users/378396/articles/494917-theassociation-between-cigarette-smoking-and-efavirenz-plasma-concentration-using-thepopulation-pharmacokinetic-approach

\section{Hosted file}

Table 3 BJCP.pdf available at https://authorea.com/users/378396/articles/494917-theassociation-between-cigarette-smoking-and-efavirenz-plasma-concentration-using-thepopulation-pharmacokinetic-approach 

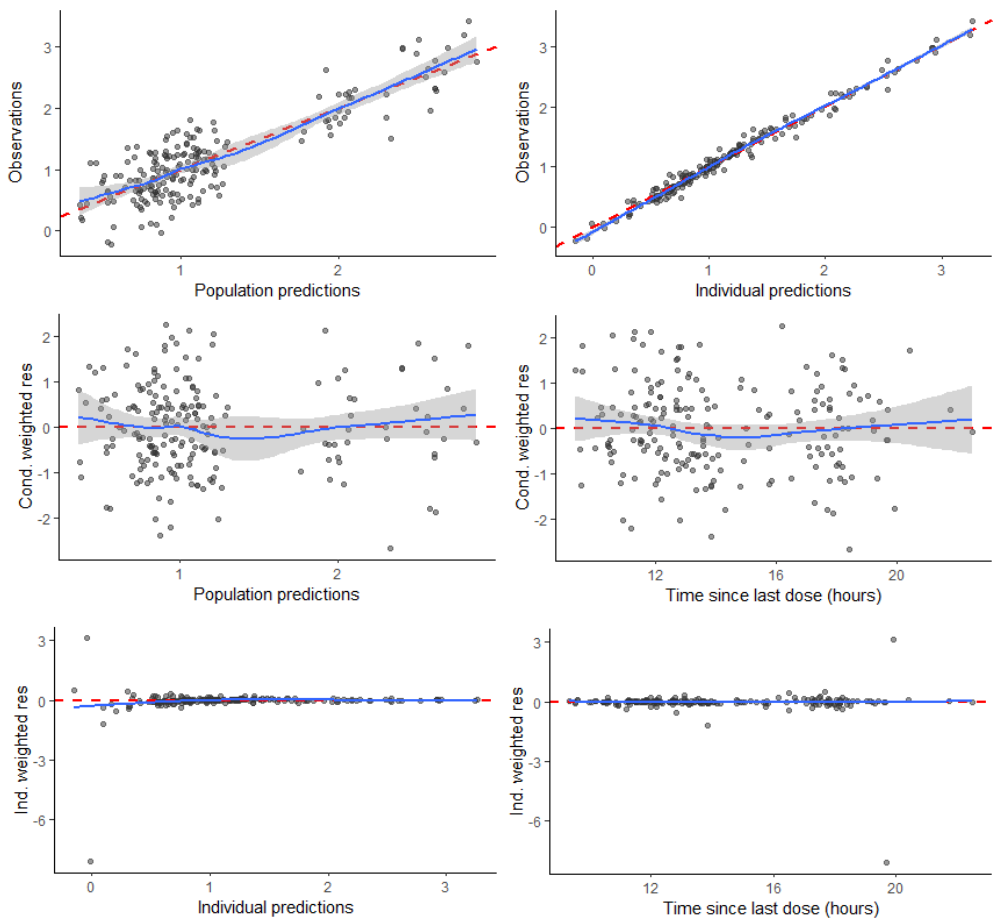

Covariate effects on efavirenz oral clearance and volume of distribution

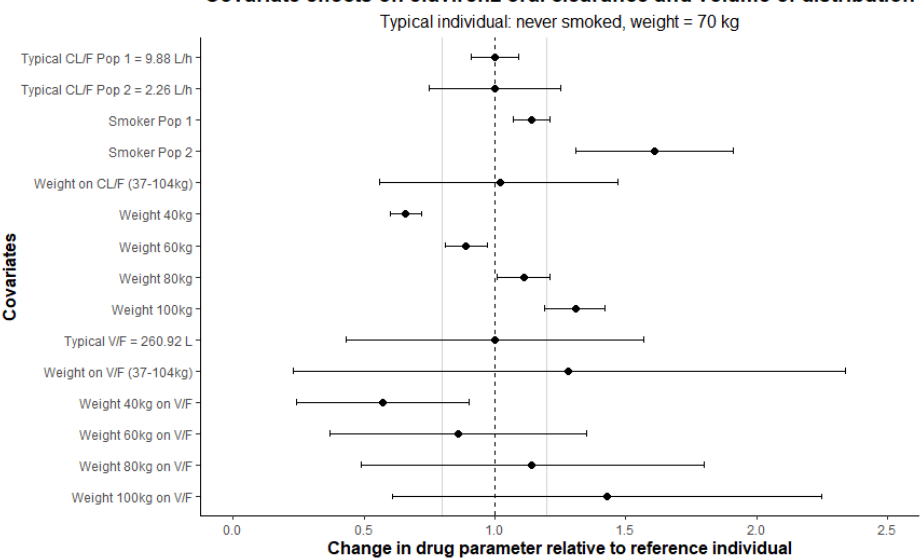



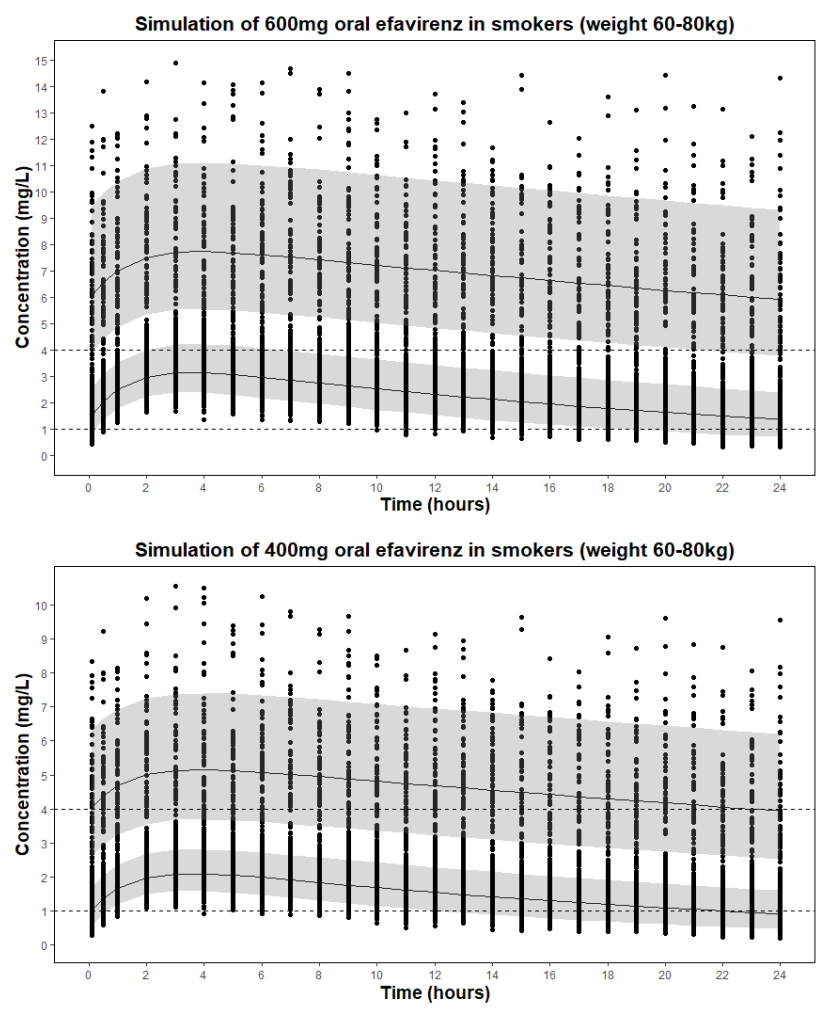

Note: The upper shaded area is the $80 \%$ prediction interval of individuals with slow $\mathrm{CL} / \mathrm{F}$, the lower shaded area is of

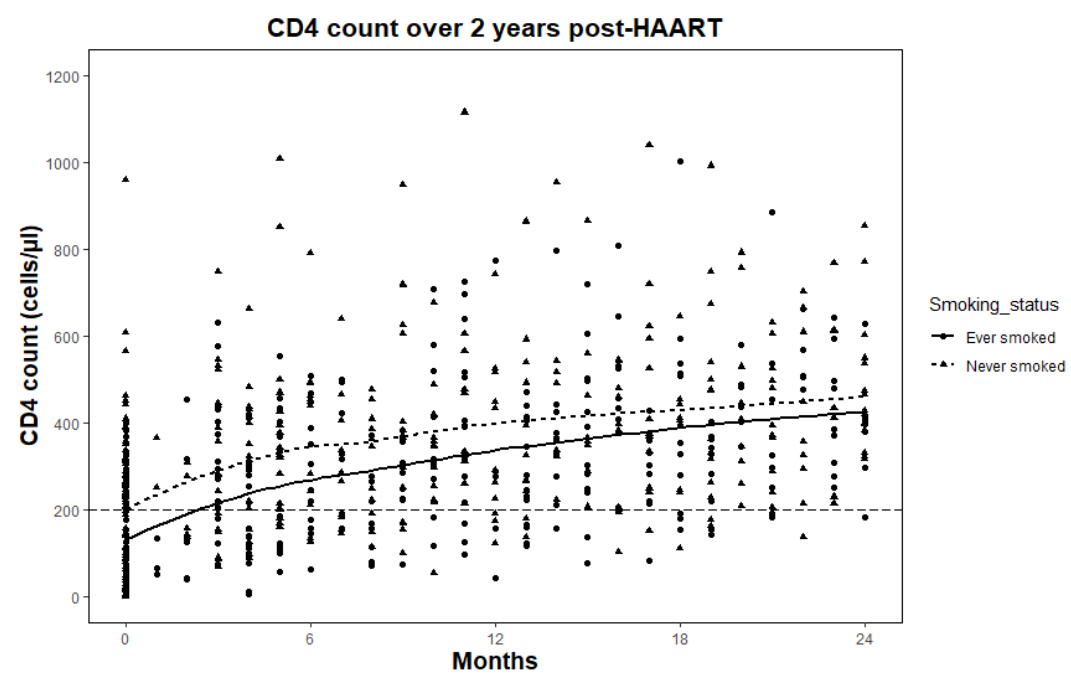




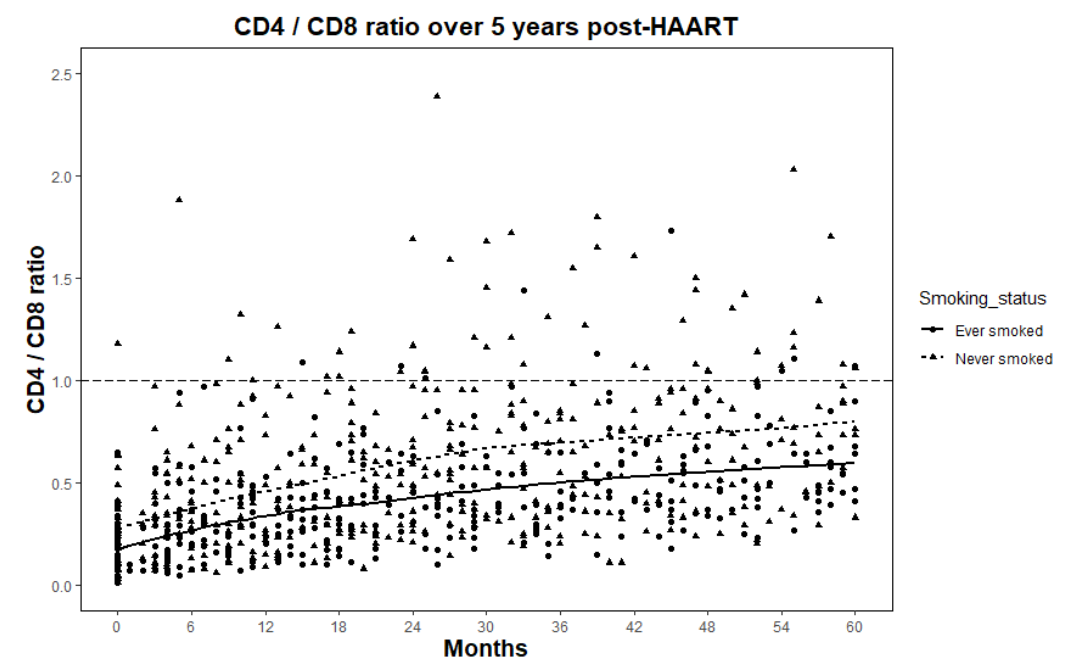

